Pacific Journal of Mathematics

ON EXTENDING ISOTOPIES 


\title{
ON EXTENDING ISOTOPIES
}

\author{
William H. Cutler
}

Let $K$ be a locally compact metric space. An isotopy on $K$ is a continuous family of homeomorphisms $h_{t}: K \rightarrow K$ for $t \in I$ such that $h_{0}=\mathrm{id}$. Let $\mathscr{F}(K)$ denote the space of isotopies of $K$ with $C-0$ topology. Conjecture: Let $X$ be metric and $Y$ a closed subset of $X$. Then every map $f: Y \rightarrow$ $\mathscr{F}(K)$ can be continuously extended to $X$. The conjecture is proved for the following cases: (1) $K$ is a 1-complex, (2) $K$ is compact and $X$ is finite-dimensional, (3) $K$ is compact, $Y$ is compact and finite-dimensional, and $X$ is separable, and (4) $Y$ is of type 1 in a compact space $X . Y$ is of type 1 in $X$ if the closure of the set of points of $X$ which do not have a unique closest point in $Y$ does not intersect $Y$.

1. Introduction. Let $K$ be a topological space. An isotopy on $K$ is a continuous family, for $t \in I$, of homeomorphisms $h_{t}: K \rightarrow K$ such that $h_{0}=\mathrm{id}$. The isotopy is invertible if $g_{t}=\left(h_{t}\right)^{-1}$ is also an isotopy (i.e., is continuous in $t$ ). Invertible isotopies can be thought of as level-preserving homeomorphisms of $K \times I$ onto itself which are the identity on $K \times\{0\}$. Throughout the paper, $K$ will be locally compact and metric, so all isotopies on $K$ will automatically be invertible. We will denote the space of isotopies on $K$ with $C-0$ topology by $\mathscr{F}(K)$. We will discuss the following:

Conjecture. Let $K$ be locally compact and metric. Let $X$ be metric and let $Y$ be a closed subset of $X$. Then every map $\varphi: Y \rightarrow$ $\mathscr{F}(K)$ can be continuously extended to $X$.

It should be noted that this conjecture is equivalent to saying that $\mathscr{F}(K)$ is an absolute retract for metric spaces. The following theorem states the conjecture for several special cases:

THEOREM. The conjecture is true in the following cases:

(1) $K$ is a one-dimensional simplicial complex (for example, $R^{1}, S^{1}$, or $\left.I\right)$

(2) $K$ is compact, $X$ is finite-dimensional

(3) $K$ is compact, $Y$ is finite-dimensional and compact, and $X$ is separable.

(4) $X$ is compact and $Y$ is of type 1 in $X$.

The last case is an easy result, the definition of type being as follows: Let $Y$ be a closed subset of $X$. Define $H(Y)=\{x \in X \mid x$ does not have a unique closest point in $Y\}$. Let $Y_{1}=\overline{H(Y)} \cap Y, Y_{n}=$ $Y_{n-1} \cap \overline{H\left(Y_{n-1}\right)}$ for $n=2,3, \cdots$. 
If $Y_{1}=\varnothing$, then we say $Y$ is of type 1 in $X$. If $Y_{n}=\varnothing$ but $Y_{n-1} \neq \varnothing$, we say $Y$ is of type $n$ in $X$. Finally, if all $Y_{i}$ are nonempty, we say that $Y$ is of infinite type in $X$. There is probably an inductive argument to show that sets of finite type in compact spaces satisfy the conjecture, but sets of infinite type are not uncommon, for instance, the Cantor set in $I$.

The proof of Part (4) of the Theorem is contained in $\S 2$. The proofs of (1)-(3) are similar to the proof of the Tietze Extension Theorem, an idea suggested to the author by R. D. Anderson, and are contained in later sections.

2. Proof of Part (4). Let $\varphi: Y \rightarrow \mathscr{F}(K)$ be continuous. Let $U$ be an open set containing $H(Y)$ such that $U \cap Y=\varnothing$. Let $f: X \rightarrow I$ be a continuous function such that $f(\bar{U})=0$ and $f(Y)=1$. If $h_{t}: K \rightarrow K$ is an isotopy, let ${ }_{s} h_{t}: K \rightarrow K$ for $s \in I$ be the isotopy defined by

$$
\begin{aligned}
& { }_{s} h_{t}=h_{t} \quad \text { for } \quad t \leqq s \\
& { }_{s} h_{t}=h_{s} \text { for } \quad t \geqq s .
\end{aligned}
$$

Define $g: X-\overline{H(Y)} \rightarrow Y$ by $g(x)$ is the unique closest point of $Y$ to $x$. Using compactness of $X$, it is easy to show that $g$ is continuous.

Finally, define $\Phi: X \rightarrow \mathscr{J}(K)$ by $\Phi(x)={ }_{f(x)} \varphi(g(x))_{t}$ on $X-\overline{H(Y)}$, and $\Phi(x)$ is the identity isotopy on $\overline{H(Y)}$. $\Phi$ is easily seen to be continuous and equals $\varphi$ on $Y$.

3. Proof of Part (1). Let $\varphi: Y \rightarrow \mathscr{J}(K)$ be continuous, where $K$ is a 1-complex. Let $K^{\prime}$ be the set of vertices of $K$ which do not intersect exactly two 1-simplices. Then any isotopy of $K$ is fixed on $K^{\prime}$. Also, $K-K^{\prime}$ is the disjoint union of sets homeomorphic to $R^{1}$ or $S^{1}$. Hence we can restrict ourselves to isotopies on these spaces. Furthermore, $\mathscr{I}\left(R^{1}\right)$ is naturally homeomorphic to $\mathscr{F}(I)$. We will prove Part (1) for $K=I$, and it will be clear that the proof generalizes to $K=S^{1}$ by the covering space of $S^{1}$, and hence to $K$ a 1-complex.

Let $f_{i} \in \mathscr{F}(I)$ for $i=1,2, \cdots, n$, and let $s_{i} \in I$ be such that $\sum_{1}^{n} s_{i}=1$. Define the $s_{i}$-average of the isotopies ${ }_{i} f_{t}$ to be the isotopy $f_{t}(x)=\sum_{i=1}^{n} s_{i i} f_{t}(x)$ for $x \in I, t \in I$. It is easy to see that $f_{t}(x)$ is an isotopy, and furthermore, that

$$
\max _{i \leqq n} d\left(f_{t},{ }_{i} f_{t}\right) \leqq \max _{i, j \leqq n} d\left({ }_{i} f_{t},{ }_{j} f_{t}\right) .
$$

Here $d$ denotes the sup metric on $\mathscr{F}(K)$ inherited from $K$ since $K$ is compact. It will also be used to denote the metric on $X$. 
We will inductively define continuous functions $\varphi_{n}: X \rightarrow \mathscr{I}(K)$ satisfying:

(a) for $y \in Y, d\left(\varphi_{n}(Y), \varphi(Y)\right)<1 / 2^{n}$

(b) for $i, j \geqq n, x \in X$ such that $d(x, Y) \geqq 1 / n$, then $\varphi_{j}(x)=\varphi_{j}(x)$

(c) $d\left(\varphi_{n}, \varphi_{n-1}\right)<1 / 2^{n-1}$.

If we can do the above, then $\varphi=\lim _{n \rightarrow \infty} \varphi_{n}$ is the required extension. It is equal to $\varphi$ on $Y$ by (a), it converges on $X-Y$ by (b), and it is continuous on $Y$ by (c).

Suppose we have inductively defined the $\varphi_{i}$ satisfying the above for $i \leqq n-1$. We will define $\varphi_{n}$ as follows.

Let $\Phi_{n}: Y \rightarrow \mathscr{J}(K)$ be defined by $\Phi_{n}(y)=\varphi(y) \circ\left(\varphi_{n-1}(y)\right)^{-1}$. (It is understood that the composition of isotopies is at each level the composition of the homeomorphisms at the same level.) Then $d\left(\Phi_{n}(y), \mathrm{id}\right)=d\left(\varphi(y), \varphi_{n-1}(y)\right)<1 / 2^{n-1}$ by (a).

Let $g: Y \rightarrow(0,1]$ be a continuous function such that if given $y_{1}, y_{2} \in Y$ and $x \in X$ such that $d\left(y_{1}, x\right)<g\left(y_{1}\right)$ and $d\left(y_{2}, x\right)<g\left(y_{2}\right)$, then $d\left(\Phi_{n}\left(y_{1}\right), \Phi_{n}\left(y_{2}\right)\right)<1 / 2^{n}$. Such a $g$ is easily constructed using continuity of $\Phi_{n}$.

Let $N_{\hat{o}}(A)$ denote the open $\delta$-neighborhood of the set $A$. Let $\mathscr{C}=\left\{N_{g(y)}(y) \cap N_{1 / m}(Y) \mid y \in Y\right\} \cup\{X-Y\} . \mathscr{C}$ is an open cover of $X$. Since $X$ is paracompact, let $\mathscr{C}^{\prime}$ be a locally finite refinement of $\mathscr{C}$. For each $u \in \mathscr{C}^{\prime}$, associate at element $f_{u} \in \mathscr{J}(K)$ as follows: If $u \subset X-Y$, then $f_{u}=\mathrm{id}$. If $u \subset N_{g(y)}(y)$ for some $y \in Y$, then $f_{u}=\Phi_{n}(y)$. Let $\left\{S_{u}: X \rightarrow I\right\}$ be a partition of unity of $\mathscr{C}^{\prime}$.

We will define $\varphi_{n}: X \rightarrow \mathscr{I}(K)$ as follows. Pick $x \in X$. Let $u_{1}, \cdots, u_{m}$ be the elements of $\mathscr{C}^{\prime}$ such that $S_{u_{i}}(x) \neq 0$ for $i=1, \cdots, m$.

Define $\Phi_{n}^{\prime}(x)$ to be the $s_{u_{i}}$-average of the isotopies $f_{u}$ and let $\varphi_{n}(x)=\Phi_{n}^{\prime}(x) \circ \varphi_{n-1}(x)$. By construction of the cover $\mathscr{C}^{\prime}$, if $y_{1}, y_{2} \in Y$ and $x \in X$ and $\left\{x, y_{1}\right\} \subset U_{1} \in \mathscr{C}^{\prime}$ and $\left\{x, y_{2}\right\} \subset U_{2} \in \mathscr{C}^{\prime}$, then $d\left(\Phi_{n}\left(y_{1}\right)\right.$, $\left.\Phi_{n}\left(y_{2}\right)\right)<1 / 2^{n}$, hence by $\left(^{*}\right), d\left(\Phi_{n}^{\prime}(y), \Phi_{n}(y)\right)<1 / 2^{n}$ for $y \in Y$. In addition, since $d\left(\Phi_{n}(y)\right.$, id $)<1 / 2^{n-1}$, it follows that $d\left(\Phi_{n}^{\prime}(x)\right.$, id $)<1 / 2^{n-1}$ for $x \in X$. Hence for $y \in Y, d\left(\varphi_{n}(y), \varphi(y)\right)=d\left(\Phi_{n}^{\prime}(y), \Phi_{n}(y)\right)<1 / 2^{n}$ and $d\left(\varphi_{n}, \varphi_{n-1}\right)=d\left(\Phi_{n}^{\prime}(x), \mathrm{id}\right)<1 / 2^{n-1}$. That part (b) of the inductive hypothesis also holds is trivial.

4. Composition sequences. Let $K$ be a compact matric space. A composition sequence of isotopies of $K$ is a finite collection $\left\{{ }_{i} f_{t}, s_{i}\right\}_{i \leqq n}$ of isotopies ${ }_{i} f_{t}: K \rightarrow K$ and numbers $s_{i} \in I$ such that $\sum s_{i}=1$. The composition of $\left\{f_{i}, s_{i}\right\}_{i \leqq n}$ is an isotopy $f_{t}: K \rightarrow K$ defined as follows:

Let $p_{m}=\sum_{1}^{m} s_{i}$ for $m \leqq n$.

If $p_{m} \leqq t \leqq p_{m+1}$ and $x \in K$, then

$$
f_{t}(x)={ }_{m+1} f_{t}(x) \circ_{m+1} f_{p_{m}}^{-1}(x) \circ_{m} f_{P_{m}}(x) \circ \cdots \circ_{2} f_{p_{2}}(x) \circ_{2} f_{p_{1}}^{-1}(x) \circ_{1} f_{p_{1}}(x) .
$$


We will now need several technical lemmas about composition sequences

LeMma 1. Let $\left\{f_{i}, s_{i}\right\}_{i \leqq n}$ be a composition sequence satisfying $d\left({ }_{i} f_{t}, \mathrm{id}\right)<\varepsilon$. Then the composition $f_{t}$ satisfies $d\left(f_{t}, \mathrm{id}\right)<2 n \varepsilon$.

\section{Proof. Trivial.}

Lemma 2. Let $f_{t} \in \mathscr{J}(K)$ and let $\varepsilon>0$. Then there exists a $\delta>0$ such that for $0 \leqq s \leqq t \leqq 1$, and $x, y \in K$ such that $d(x, y)<\delta$, then $d\left(f_{s}^{-1}(x), f_{s}^{-1}(y)\right)<\varepsilon, d\left(f_{t}(x), f_{t}(y)\right)<\varepsilon$ and $d\left(f_{t} \circ f_{s}^{-1}(x), f_{t} \circ f_{s}^{-1}(y)\right)<\varepsilon$.

Proof. Easy to verify using compactness of $K$ and $I$.

Lemma 3. Given $\varepsilon>0$ and $f_{t} \in \mathscr{J}(K)$. Then there exists a $\delta>0$ such that if $g_{t} \in \mathscr{\mathcal { F }}(K)$ satisfies $d\left(f_{t}, g_{t}\right)<\delta$, then $d\left(f_{t}^{-1}, g_{t}^{-1}\right)<\varepsilon$.

Proof. Again easy, using compactness of $K$ and $I$.

Lemma 4. Given $f_{t} \in \mathscr{J}(K)$, there exists a function $l:(0,1] \rightarrow$ $(0,1]$ such that for any $\varepsilon \in(0,1], g_{t} \in \mathscr{J}(K), \quad x, y \in K$ such that $d\left(f_{t}, g_{t}\right)<l(\varepsilon)$ and $d(x, y)<l(\varepsilon)$ and $0 \leqq s \leqq t \leqq 1$, then

$$
d\left(f_{t} \circ f_{s}^{-1}(x), g_{t} \circ g_{s}^{-1}(y)\right)<\varepsilon .
$$

Proof. $\quad d\left(f_{t} \circ f_{s}^{-1}(x), g_{t} \circ g_{s}^{-1}(y)\right) \leqq d\left(f_{t} \circ f_{s}^{-1}(x), f_{t} \circ f_{s}^{-1}(y)\right)+d\left(f_{t} \circ f_{s}^{-1}(y)\right.$, $\left.f_{t} \circ g_{s}^{-1}(y)\right)+d\left(f_{t} \circ g_{s}^{-1}(y), g_{t} \circ g_{s}^{-1}(y)\right)$. The first term is minimized by Lemma 2 , the second by Lemma 3 and uniform continuity of $f_{t}$, and the third is minimized by $l(\varepsilon)$.

Lemma 5. Let $f_{t} \in \mathscr{F}(K)$ and let $\varepsilon>0$ and $n$ be an integer. Then there exists $a \delta>0$ such that if $\left\{{ }_{i} g_{t}, s_{i}\right\}_{i \leqq n}$ is a composition sequence such that $d\left({ }_{i} g_{t}, f_{t}\right)<\delta$ for all $i \leqq n$, then the composition $g_{t}$ satisfies $d\left(g_{t}, f_{t}\right)<\varepsilon$.

Proof. Let $\delta=l^{n}(\varepsilon)$ (the composition of $n l s$ ) where $l$ is the function of Lemma 4 .

5. Proofs of Parts (2) and (3). We will now prove Part (2) in a fashion analogous to that of Part (1). Let $\varphi: Y \rightarrow \mathscr{I}(K)$ be continuous, where $K$ is compact and $\operatorname{dim}(x)=n-1$. We will inductively define functions $\varphi_{m}: X \rightarrow \mathscr{F}(K)$ satisfying:

(a) for $y \in Y, d\left(\varphi_{m}(y), \varphi(y)\right)<1 / 2_{m}$ 

$\varphi_{j}(x)$

(b) for $i, j \geqq m$ and $x \in X$ such that $d(x, Y) \geqq 1 / m, \varphi_{i}(x)=$

( c ) $d\left(\varphi_{m}, \varphi_{m-1}\right) \leqq 2 n / 2^{m-1}$.

Again, define $\Phi_{n}(y)=\varphi(y) \circ\left(\varphi_{n-1}(y)\right)^{-1}$.

Let $g: Y \rightarrow(0,1]$ be a function such that if $x_{1}, \cdots, x_{n}$ are points of $X$ such that $d\left(x_{i}, y\right)<g(y)$ for some $y \in Y$ and all $i \leqq n$, then any composition $f_{t}$ of the isotopies $\Phi_{n}\left(x_{i}\right)$ satisfies $d\left(f_{t}, \Phi_{n}(y)\right)<1 / 2^{n}$. Such a function is constructed using Lemma 4 and the continuity of $\Phi_{n}$.

We now take a cover $\mathscr{C}$ of $X$ as sets of the form $\left\{N_{g(y)} \cap\right.$ $\left.N_{1 / m}(Y) \mid y \in Y\right\} \cup\{X-Y\}$ as in $\S 3$. Take a refinement or order $n-1$ (see Theorem V 1, p. 48 of [2]), call it $\mathscr{C}^{\prime}$. Order the elements of $\mathscr{C}^{\prime}$, take a portion of unity, and define $\Phi_{n}^{\prime}: X \rightarrow \mathscr{I}(K)$ by using compositions instead of $s_{i}$-averages. Letting $\varphi_{n}(x)=$ $\Phi_{n}^{\prime}(x) \circ \varphi_{n-1}(x)$ as before, the inductive hypotheses are again met. Note that (c), $d\left(\varphi_{m}, \varphi_{m-1}\right) \leqq 2 n / 2^{m-1}$ follows from Lemma 1 .

Part (3) follows as a corollary of (2). Since $X$ is separable, it may be imbedded in an endslice of the Hilbert cube $Q$. Call the imbedding $i$. Since $Y$ is compact, $i(Y)$ is closed, and hence a $Z$-set in the cube. If $Y$ has dimension $n$, it may also be imbedded in $I^{2 n+1}$, call the imbedding $j$. Then $i(Y)$ and $j(Y)$ are $Z$-sets in the cube. By [1] there exists a homeomorphism $h: Q \rightarrow I^{2 n+1} \times Q$ such that $h \mid i(Y)$ is onto $j(Y) \times\{0\}$.

By Part (2), we can extend the map $\varphi \circ(h \circ i)^{-1}$ on $(h \circ i)(Y)$ to $I^{2 n+1} \times\{0\}$. There is then a natural extension to $I^{2 n+1} \times Q$ and hence to $(h \circ i)(x)$.

\section{REFERENCES}

1. R. D. Anderson, On topological infinite deficiency, Mich. Math. J., 14 (1967), 365-383.

2. Hurewicz and Wallman, Dimension Theory, Princeton University Press, 1948.

Received February 7, 1972 and in revised form May 24, 1972,

Louisiana State UNIVERSity 



\section{PACIFIC JOURNAL OF MATHEMATICS}

\section{EDITORS}

D. Gilbarg and J. Milgram Stanford University

Stanford, California 94305

R. A. Beaumont

University of Washington

Seattle, Washington 98105
J. DugundJI

Department of Mathematics

University of Southern California

Los Angeles, California 90007

RICHARD ARENS

University of California

Los Angeles, California 90024

ASSOCIATE EDITORS
E. F. BECKENBACH
B. H. NeumanN
F. WOLF
K. YoSHIDA

\section{SUPPORTING INSTITUTIONS}

UNIVERSITY OF BRITISH COLUMBIA

CALIFORNIA INSTITUTE OF TECHNOLOGY

UNIVERSITY OF CALIFORNIA

MONTANA STATE UNIVERSITY

UNIVERSITY OF NEVADA

NEW MEXICO STATE UNIVERSITY

OREGON STATE UNIVERSITY

UNIVERSITY OF OREGON

OSAKA UNIVERSITY
UNIVERSITY OF SOUTHERN CALIFORNIA

STANFORD UNIVERSITY

UNIVERSITY OF TOKYO

UNIVERSITY OF UTAH

WASHINGTON STATE UNIVERSITY

UNIVERSITY OF WASHINGTON

$\stackrel{*}{*} \stackrel{*}{*} \stackrel{*}{*}$ AMERICAN MATHEMATICAL SOCIETY

NAVAL WEAPONS CENTER 


\section{Pacific Journal of Mathematics}

\section{Vol. 46, No. $1 \quad$ November, 1973}

Allan Francis Abrahamse, Uniform integrability of derivatives on $\sigma$-lattices .......................................... 1

Ronald Alter and K. K. Kubota, The diophantine equation $x^{2}+D=p^{n} \ldots \ldots \quad 11$

Grahame Bennett, Some inclusion theorems for sequence spaces .......... 17

William Cutler, On extending isotopies ........................ 31

Robert Jay Daverman, Factored codimension one cells in Euclidean

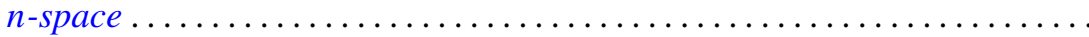

Patrick Barry Eberlein and Barrett O’Neill, Visibility manifolds ............ 45

M. Edelstein, Concerning dentability .......................... 111

Edward Graham Evans, Jr., Krull-Schmidt and cancellation over local

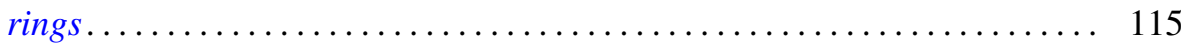

C. D. Feustel, A generalization of Kneser's conjecture ................ 123

Avner Friedman, Uniqueness for the Cauchy problem for degenerate parabolic equations .......................................... 131

David Golber, The cohomological description of a torus action ............ 149

Alain Goullet de Rugy, Un théorème du genre "Andô-Edwards" pour les

Fréchet ordonnés normaux..............................

Louise Hay, The class of recursively enumerable subsets of a recursively enumerable set ........................................

John Paul Helm, Albert Ronald da Silva Meyer and Paul Ruel Young, On orders of translations and enumerations...

Julien O. Hennefeld, A decomposition for $B(X)^{*}$ and unique Hahn-Banach

extensions

Gordon G. Johnson, Moment sequences in Hilbert space .

Thomas Rollin Kramer, A note on countably subparacompact spaces ...

Yves A. Lequain, Differential simplicity and extensions of a derivation ....

Peter Lorimer, A property of the groups Aut $\mathrm{PU}\left(3, q^{2}\right) \ldots$

225

Yasou Matsugu, The Levi problem for a product manifold.

231

John M.F. O'Connell, Real parts of uniform algebras ......

William Lindall Paschke, A factorable Banach algebra without bounded

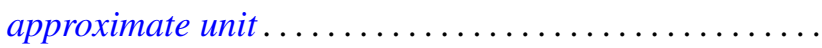

Ronald Joel Rudman, On the fundamental unit of a purely cubic field ....

Tsuan Wu Ting, Torsional rigidities in the elastic-plastic torsion of simply connected cylindrical bars .........................

Philip C. Tonne, Matrix representations for linear transformations on analytic sequences...................................

Jung-Hsien Tsai, On E-compact spaces and generalizations of perfect

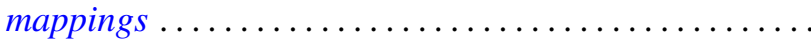

Alfons Van Daele, The upper envelope of invariant functionals majorized by an invariant weight. .. 